\title{
Guest Editorial: Special Issue on Algorithms and Computation
}

\author{
Kyung-Yong Chwa · Kunsoo Park
}

Received: 20 June 2012 / Accepted: 6 July 2012 / Published online: 3 August 2012

(C) Springer Science+Business Media, LLC 2012

This special issue is dedicated to the themes of Algorithms and Computation. The eight papers in this issue cover a wide range of areas within the themes such as graph partitioning, graph matching, graph coloring, tree partitioning, graph minor theory, polynomial multiplication, algorithmic self-assembly, and the complexity of counting problems.

The paper "Beyond good partition shapes: an analysis of diffusive graph partitioning" by Meyerhenke and Sauerwald (doi:10.1007/s00453-012-9666-y) studies the problem of graph partitioning and gives a theoretical analysis of the diffusionbased partitioning heuristic Bubble-FOS/C which yields good experimental results in practice.

In the paper "Improved algorithms for even factors and square-free simple bmatchings" (doi:10.1007/s00453-012-9642-6), Babenko considers the problem of finding an even factor of maximum cardinality, which is a generalization of path matchings. He gives an improved algorithm to find a maximum cardinality even factor in an odd-cycle symmetric digraph.

The paper "3-coloring AT-free graphs in polynomial time" by Stacho (doi:10.1007/ s00453-012-9624-8) deals with graph coloring and gives a polynomial time algorithm for the 3-coloring problem of asteroidal-triple-free graphs.

In "Minimum cost partitions of trees with supply and demand" (doi:10.1007/ s00453-011-9573-7), Ito, Hara, Zhou and Nishizeki consider the problem of parti-

K.-Y. Chwa

Department of Computer Science, Korea Advanced Institute of Science and Technology, Daejeon, Korea

e-mail: kychwa@jupiter.kaist.ac.kr

K. Park $(\bowtie)$

Department of Computer Science and Engineering, Seoul National University, Seoul, Korea e-mail: kpark@snu.ac.kr 
tioning a tree with supply vertices and demand vertices into subtrees with minimum cost. They show that the problem is NP-hard and give a fully polynomial-time approximation scheme for the problem.

In "Improved bounds on the planar branchwidth with respect to the largest grid minor size" (doi:10.1007/s00453-012-9627-5), Gu and Tamaki study the relationship between the branchwidth and the grid minor of a graph. They present improved bounds in the relationship for planar graphs.

The paper "Computing sparse multiples of polynomials" by Giesbrecht, Roche and Tilak (doi:10.1007/s00453-012-9652-4) considers the problem of finding a sparse multiple of a polynomial over a field. When the field is of rational numbers, it gives a polynomial time algorithm, and for a finite field, it shows that the problem is at least as hard as determining the multiplicative order of elements in an extension field.

The paper "Identifying shapes using self-assembly" by Patitz and Summers (doi:10.1007/s00453-011-9549-7) introduces a problem in algorithmic self-assembly: Given an input shape as the seed, design a finite tile set that can uniquely identify whether or not the given input shape matches a particular target shape. It studies the complexities of identifying squares and some more general shapes.

In "From Holant to \#CSP and back: dichotomy for Holant problems" (doi:10. 1007/s00453-012-9626-6), Cai, Huang and Lu explore the relationship between two frameworks, counting Constraint Satisfaction Problems (\#CSP) and Holant Problems, to study the complexity of counting problems. They show dichotomy theorems for some Holant problems.

All the papers in this issue went through the rigorous reviewing process of Algorithmica. The guest editors are grateful to authors and reviewers for contributing to this special issue. 